Supporting Information

\title{
Direct Iodination of Alkanes
}

Raúl Montoro and Thomas Wirth*

Department of Chemistry, Cardiff University, P.O. Box 912, Cardiff CF10 3TB, U.K. wirth@cf.ac.uk

Representative procedure: Iodocyclohexane 9a: Iodine (5.08 g, $20 \mathrm{mmol})$ was dissolved in cyclohexane $(220 \mathrm{~mL}, 2 \mathrm{~mol})$ and sodium tert-butoxide $(1.92 \mathrm{~g}, 20 \mathrm{mmol})$ was added. The suspension was stirred at $40{ }^{\circ} \mathrm{C}$ for $15 \mathrm{~h}$. The mixture was washed with $0.2 \%$ aq. $\mathrm{Na}_{2} \mathrm{~S}_{2} \mathrm{O}_{3}(60 \mathrm{~mL})$ and dried over $\mathrm{MgSO}_{4}$. The solvent was removed under reduced pressure and the residue distilled to yield iodocyclohexane 9a (3.55 g, 84\%) as a colorless liquid. All reaction products were identified by GC/MS and NMR analysis and were found to be identical to published data.

Spectral data of the iodoalkanes prepared can be found in the following references:

1-Iodopentane 5a

Dostovalova, V. I.; Velichko, F. K.; Fridlina, R. Kh. Bull. Acad. Sci. USSR Div. Chem. Sci. 1987, 36, 701.

2-Iodopentane $\mathbf{5 b}$

Ejchart, A. Org. Magn. Reson. 1981, 15, 22.

3-Iodopentane 5c

Wiberg, K. B.; Pratt, W. E.; Bailey, W. F. J. Org. Chem. 1980, 45, 4936.

1-Iodohexane 6a

Tiers, G. V. D. Magn. Reson. Chem. 1999, 37, 609.

2-Iodohexane $\mathbf{6 b}$

3-Iodohexane 6c

Ejchart, A. Org. Magn. Reson. 1981, 15, 22.

1-Iodoheptane 7a

Reddy, C. K.; Periasamy, M. Tetrahedron 1992, 48, 8329.

2-Iodoheptane $\mathbf{7 b}$

3-Iodoheptane 7c

Dostovalova, V. I.; Velichko, F. K.; Fridlina, R. Kh. Bull. Acad. Sci. USSR Div. Chem. Sci. 1987, 36, 701.

Iodocyclopentane 8a

Wiberg, K. B.; Pratt, W. E.; Bailey, W. F. J. Org. Chem. 1980, 45, 4936.

Iodocyclohexane 9a

Inoue, Y.; Fukunaga, T.; Hakushi, T. J. Org. Chem. 1983, 48, 1732.

Wiberg, K. B.; Pratt, W. E.; Bailey, W. F. J. Org. Chem. 1980, 45, 4936. 
Iodocycloheptane 10a

Joseph, R.; Pallan, S. P.; Sudalai, A.; Ravindranathan, T. Tetrahedron Lett. 1995, 36, 609.

Benzyliodide 11a

Davies, S. G.; Smyth, D. G.; Chippindale, A. M. J. Chem. Soc., Perkin Trans. 1 1999, 3089. 